\title{
Inflammatory and Humoral Immune Status in Chronic Kidney Disease
}

\author{
Deepika L Yarlagadda ${ }^{1}$, Pradeep Japa ${ }^{2}$, Mohammed Noorjahan ${ }^{3}$, Sai Baba KSS ${ }^{4}$, Sreedevi NN ${ }^{5}$, Sree Bhushan Raju ${ }^{6}$
}

\begin{abstract}
Background: Chronic kidney disease (CKD) is a present world health problem. It is widely accepted that uremia, depresses the immunity and make them most susceptible to infection. The relationship between uremia, impaired immune status, and susceptibility to infection has never been established.

Aim: The study was designed to know the association between levels of immunoglobulins and inflammatory marker hs-CRP in various stages of CKD patients and comparing it with healthy controls.
\end{abstract}

Materials and methods: One hundred twenty-one CKD patients staged based on eGFR were evaluated for serum Immunoglobulins (G, A\&M), hs-CRP and compared between the different stages and also with healthy controls $(n=24)$.

Results: Out of $121 \mathrm{CKD}$ patients (male $=88$, female $=33$ ) with a mean age of $46 \pm 15$ years, known cases of hypertension (HTN) were seen in $32 \%$, both DM and HTN 38\%, DM only in $1 \%$ and remaining were with no h/o DM or HTN. The median levels of blood urea, serum creatinine, and hs-CRP are significantly high across the CKD stages and when compared to healthy controls $(p<0.0001)$. IgM levels showed significant $(p=0.0005)$ decrease across all the stages of CKD, whereas IgG levels have decreased trend across the stages but statistically not significant.

Conclusion: Based on our findings it can be concluded that a deficiency of immunoglobulins was noticed in a considerable number of uremic patients from all stages of CKD, suggesting inhibition of their synthesis by the uremic state. Uremic patient is associated with a state of immune dysfunction characterized by immunodepression that leads to a high prevalence of infections, and also by immunoactivation causing inflammation (increase in hsCRP).

Keywords: CKD, Immunoglobulins, hs CRP, Inflammation.

Indian Journal of Medical Biochemistry (2019): 10.5005/jp-journals-10054-0108

\section{INTRODUCTION}

Chronic kidney disease (CKD) is a present world health problem. CKD is defined as either renal damage or GFR $<60 \mathrm{~mL} / \mathrm{min} / 1.73 \mathrm{~m}^{2}$ for more than or equal to 3 months. CKD is defined as abnormalities of kidney structure or function, occurring for more than 3 months, with implications for health. ${ }^{1}$ The incidence of CKD is increasing day by day as a result of growing elderly population. ${ }^{2}$ According to the Indian society of nephrology, 1 in 10 persons in the general population are suffering from some form of CKD. About 175,000 new people have kidney failure (stage 5 CKD) every year in India and require dialysis or kidney transplantation. ${ }^{3}$ The two common causes of CKD are Diabetes and Hypertension which are responsible for more than $50 \%$ cases, and these diseases are increasing rapidly in Indian patients. Infectious diseases are the second most common causes of morbidity and mortality after cardiovascular disease in CKD patients, contributing to $30-36 \%$ of deaths among patients on dialysis. ${ }^{4}$ Uremic toxins, malnutrition, and immunosuppressive medications are the factors leading to immune dysregulation, which are further complicated by renal replacement therapies. ${ }^{5}$

Both innate and adaptive immune systems are affected in patients with end-stage renal disease (ESRD). Anti-inflammatory interleukin (IL-10) and pro-inflammatory cytokines like tumor necrosis factor- $a$ (TNF- $a$ ) and as well as interleukin- 6 (IL-6) are also increased. Cytokine accumulation occurs as a consequence of decreased renal clearance and increased production. ${ }^{6}$

There is firm evidence that irrespective of the cause of renal disease an acute and chronic pro-inflammatory state exists which leads to inflammation contributing to morbidity and mortality in adults with CKD and ESRD. Inflammatory cascade may simultaneously be stimulated by any disease process causing renal injury. ${ }^{8}$

\footnotetext{
1,2Junior Resident, ${ }^{3}$ Associate Professor, ${ }^{4,6}$ Professor and Head,

${ }^{5}$ Assistant Professor,

${ }^{1-5}$ Department of Biochemistry, Nizam's Institute of Medical Sciences, Hyderabad, Telangana, India

${ }^{6}$ Department of Nephrology, Nizam's Institute of Medical Sciences, Hyderabad, Telangana, India
}

Corresponding Author: Mohammed Noorjahan, Associate Professor, Department of Biochemistry, Nizam's Institute of Medical Sciences, Hyderabad, Telangana, India, Phone: 09440207335 e-mail: m_noorjahan@yahoo.co.in How to cite this article: Yarlagadda DL, Japa P, Noorjahan M, Baba SKSS, Sreedevi NN, Raju SB. Inflammatory and Humoral Immune Status in Chronic Kidney Disease. Indian J Med Biochem 2019;23(2):303-307.

Source of support: Nil

Conflict of interest: None

Even though some compensatory mechanisms exist to protect the individual with kidney disease from unabated inflammation, the balance favors the progression of kidney disease. ${ }^{8}$ Understanding of the mechanisms mediating endothelial dysfunction and inflammation is necessary to combat the destructive effects of inflammation in CKD and ESRD patients. ${ }^{9}$

The present study was undertaken because of the paucity of humoral immune status studies in CKD patients and to emphasize the role of the immune dysfunction and the inflammation in CKD.

\section{AIm}

The study was designed to investigate the changes in immunoglobulins and the inflammatory marker hs-CRP in various stages of CKD patients and compare it with healthy controls.

(O) The Author(s). 2019 Open Access This article is distributed under the terms of the Creative Commons Attribution 4.0 International License (https://creativecommons. org/licenses/by-nc/4.0/), which permits unrestricted use, distribution, and non-commercial reproduction in any medium, provided you give appropriate credit to the original author(s) and the source, provide a link to the Creative Commons license, and indicate if changes were made. The Creative Commons Public Domain Dedication waiver (http://creativecommons.org/publicdomain/zero/1.0/) applies to the data made available in this article, unless otherwise stated. 


\section{Materials and Methods}

It is a retrospective study of predialysis CKD patients from inpatient ward of Nephrology Department, Nizam's Institute of Medical Sciences. All the subjects are diagnosed as CKD, previously by clinical, biochemical and histopathological characteristics. Exclusion criteria were age below 18 years, chronic hepatic disease. Biochemical parameters analyzed were serum creatinine, serum Immunoglobulin $\mathrm{G}(\lg G)$, serum Immunoglobulin A ( $\lg A)$, serum Immunoglobulin $M(\operatorname{Ig} M)$ and serum high sensitive $C$ reactive protein (hs-CRP). Methods used to estimate are kinetic Jaffe method for sr.creatinine with IDMS traceable calibrator, immunoturbidimetry method for serum immunoglobulins and serum hs-CRP on autoanalyzer Beckman AU480. eGFR calculated for all the patients using Modification of Diet in Renal Disease (MDRD) 4 variable formula using age, gender, race, and serum Creatinine. CKD patients were divided into CKD stages according to eGFR. Stage 3 is eGFR $\left(\mathrm{mL} / \mathrm{min} / 1.73 \mathrm{~m}^{2}\right)$ between 30 to 59 , in stage 4 eGFR between 15 to 29 and stage 5 is eGFR of less than $15 \mathrm{~mL} / \mathrm{min} / 1.73 \mathrm{~m}^{2}$ or end-stage renal disease(ESRD).

\section{Statistical Calculations}

Statistical analysis was done using Prism 7 (Graph Pad Software Inc). Distribution of normality was established by the Shapiro-Wilk normality test. Results were expressed as the median and interquartile range (IQR). Mann-Whitney $\mathrm{U}$ and Kruskal-Wallis tests are used to determine statistical significance between the groups. $p<0.05$ is considered as statistically significant. The correlation was done by using Spearman correlation analysis.

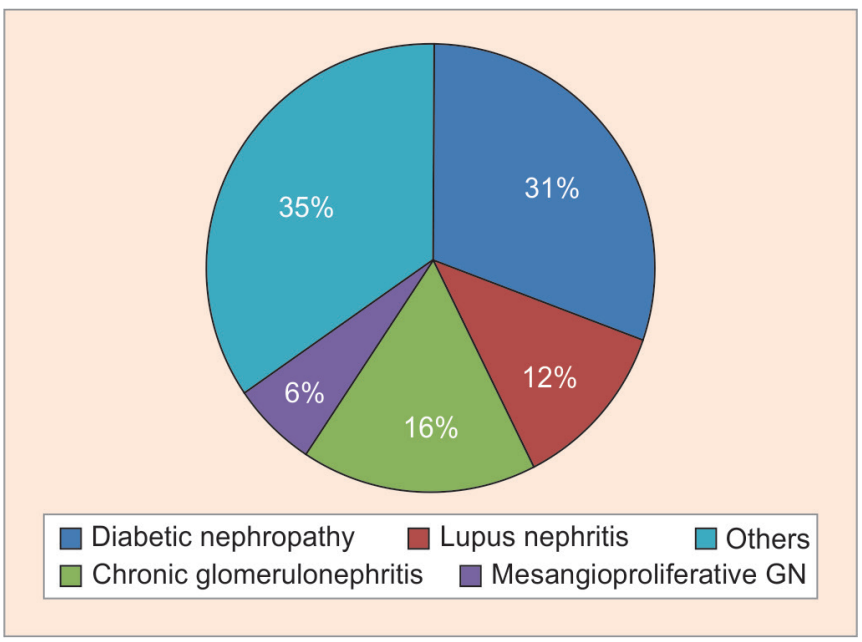

Fig. 1: Showing various etiologies of CKD patients

\section{Results}

Our study enrolled 121 patients (male $=88$, female $=33$ ) with mean age of $46 \pm 15 y$ rs, which included 3, 4 and 5 stages of CKD patients with various etiologies (Fig. 1) and 24 healthy controls. Out of 121 patients, known cases of hypertension (HTN) were 32\%, DM only in $1 \%$, both DM and HTN in $38 \%$ and remaining were with no h/o DM or HTN. Based on eGFR maximum number of people, i.e., $41 \%$ $(n=50)$ fall in stage 5 and $32 \%(n=39)$ and $27 \%(n=31)$ in stage 4 and stage 3 , respectively (Fig. 2).

As seen in Table 1, median (IQR) values of Blood urea (mg/dL) in CKD patients and controls were 95 (66-122)and 20 (16-23) respectively which was significantly high in CKD $(p<0.0001)$ compared to controls. Similarly creatinine also showed significant increase in CKD patients. Median (IQR) values of sr.creatinine $(\mathrm{mg} / \mathrm{dL})$ in CKD patients and Controls were 4.4 (2.4-6.4)and 0.9 (0.7-1.1) with $p<0.0001$, respectively. CKD patients have shown significant decrease in immunoglobulins (mg/dL) IgG [1024 (795-1271) vs 1213 (1060-1329), $p=0.0177]$, and IgM [55 (40-88) vs. 115 (75-135), $p<0.0001$ ] when compared to healthy control group (Fig. 3). Though there was no significant difference in IGA [205 (158-295) vs 216 (179-293), $\underline{p}=0.495]$, the levels were lower in cases compared to controls. hs-CRP $(\mathrm{mg} / \mathrm{L})$ in cases showed significant increase [19.8 (3.5-60.5) vs $1.7(0.9-2.9), p<0.0001]$ compared to control group (Fig. 4).

The median levels of blood urea, sr.creatinine and hs-CRP (Table 2) are significantly higher in across the CKD stages and when compared to healthy controls ( $p<0.0001)$. There were a significant decrease in $\lg M(p=0.0005)$ levels across all the stages of CKD whereas IgG levels have shown decreased trend across

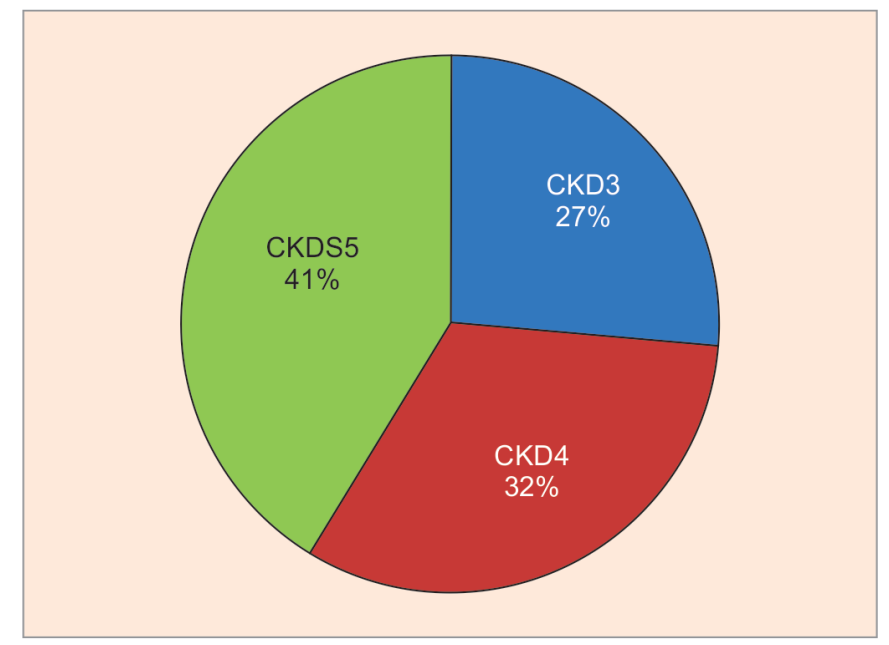

Fig 2: Stage wise distribution of CKD patients

Table 1: Biochemical parameters in Controls and Cases:

\begin{tabular}{llll}
\hline Parameters & Controls median $(I Q R)$ & Cases median $(I Q R)$ & $p$ value \\
\hline Blood urea $(\mathrm{mg} / \mathrm{dL})$ & $20(16-23)$ & $95(66-122)$ & $<0.0001^{*}$ \\
Sr. Creatinine $(\mathrm{mg} / \mathrm{dL})$ & $0.9(0.7-1.1)$ & $4.4(2.45-6.45)$ & $<0.0001^{*}$ \\
Sr. hsCRP $(\mathrm{mg} / \mathrm{L})$ & $1.7(0.95-2.93)$ & $19.85(3.5--60.5)$ & $<0.0001^{*}$ \\
Sr. $\operatorname{lgG}(\mathrm{mg} / \mathrm{dL})$ & $1213(1060-1329)$ & $1024(795.5-1271)$ & $0.0177^{*}$ \\
Sr. $\operatorname{lgA}(\mathrm{mg} / \mathrm{dL})$ & $216.5(179.3-292.8)$ & $205(158.5-295.5)$ & 0.495 \\
Sr. $\operatorname{lgM}(\mathrm{mg} / \mathrm{dL})$ & $115.5(75.5-135.3)$ & $55(40-88.5)$ & $<0.0001^{*}$ \\
\hline
\end{tabular}

${ }^{*} p<0.05$ is considered significant, IQR-interquartile range 


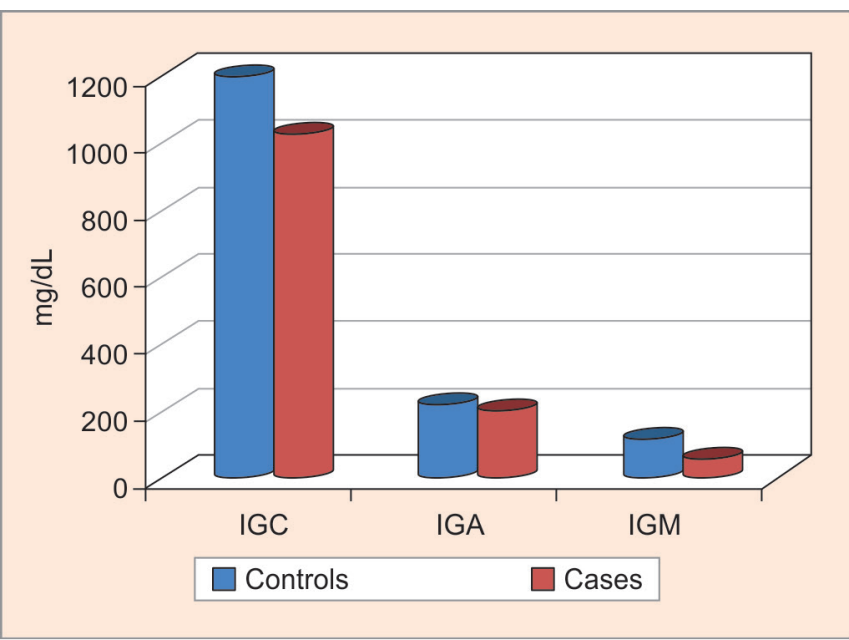

Fig 3: Median values of S. immunoglobulins in controls and CKD cases

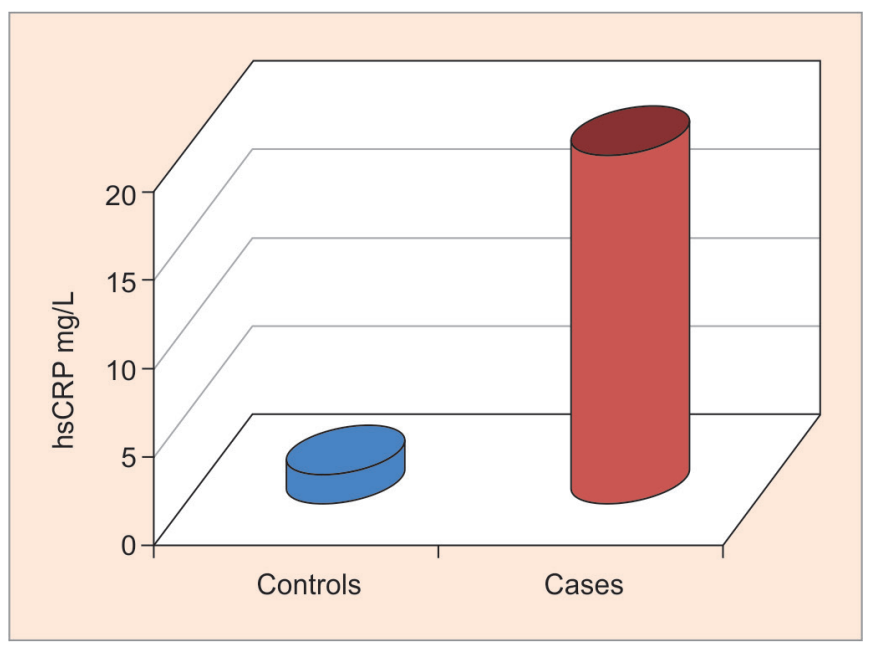

Fig 4: Median values of S. hsCRP in controls and CKD cases

Table 2: Investigations in controls and various stages of CKD Patients.

\begin{tabular}{|c|c|c|c|c|c|}
\hline Parameters & $\begin{array}{l}\text { Controls }(n=24) \\
\text { median }(I Q R)\end{array}$ & $\begin{array}{l}\text { Stage } 3(n=31) \\
\text { median }(I Q R)\end{array}$ & $\begin{array}{l}\text { Stage } 4(n=39) \\
\text { median }(I Q R)\end{array}$ & $\begin{array}{l}\text { Stage } 5(n=50) \\
\text { median }(I Q R)\end{array}$ & $p$ value \\
\hline Blood Urea (mg/dL) & $20(16-23)$ & $56(44-79)$ & $89(69-113)$ & $119(96-150)$ & $<0.0001^{*}$ \\
\hline eGFR $\left(\mathrm{mL} / \mathrm{min} / \mathrm{m}^{2}\right)$ & 105 (92-119) & $37(32-44.7)$ & $20(17-25)$ & $9(7-11)$ & $<0.0001^{*}$ \\
\hline S. creatinine $(\mathrm{mg} / \mathrm{dL})$ & $0.9(0.7-1.1)$ & $2.05(1.8-2.3)$ & $3.9(3.1-4.5)$ & $7.15(5.8-9.7)$ & $<0.0001^{*}$ \\
\hline S. hs-CRP (mg/L) & $1.7(0.95-2.93)$ & $3.6(0.95-9.0)$ & $20(4.65-72.0)$ & $30.2(5.5-99)$ & $<0.0001^{*}$ \\
\hline S. $\lg G(\mathrm{mg} / \mathrm{dL})$ & 1213(1060-1329) & $1145(871-1436)$ & $992(836-1223)$ & $968(742-1206)$ & 0.2719 \\
\hline S. $\lg A(m g / d L)$ & $216.5(179-293)$ & $188(157-247)$ & $222(160-329)$ & $202(158-295)$ & 0.5735 \\
\hline S. $\operatorname{lgM}(\mathrm{mg} / \mathrm{dL})$ & $115.5(75.5-135)$ & $77.5(61-117.8)$ & $52(33-84)$ & $50(38.2-66.7)$ & $0.0005^{*}$ \\
\hline
\end{tabular}

${ }^{*} p<0.05$ is considered significant; IQR, interquartile range

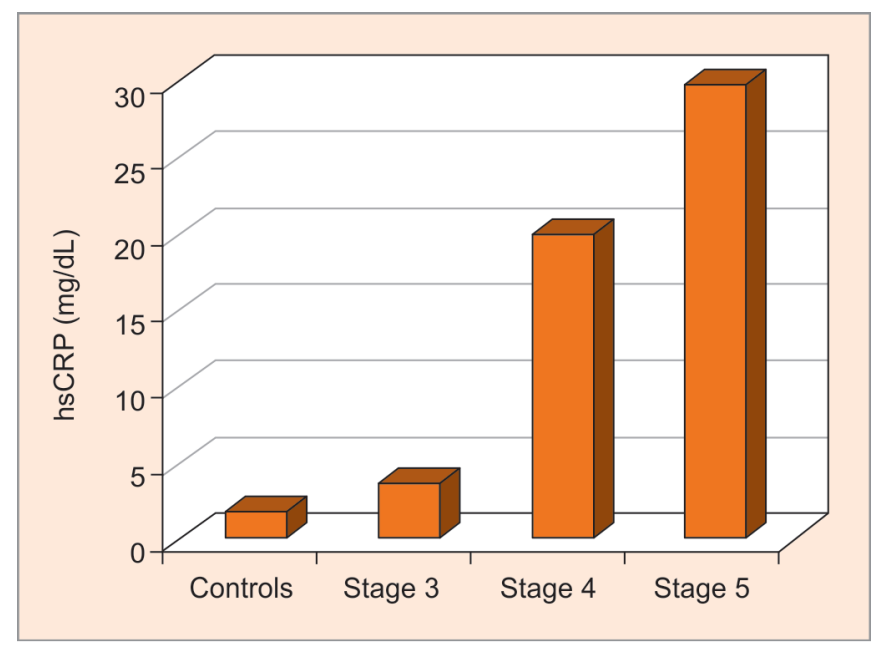

Fig. 5: Comparison of hs-CRP in controls and CKD stages

the stages but statistically not significant. Immunoglobulin A levels did not show a statistically significant difference between the stages and with controls. Figures 5 and 6 demonstrate that as CKD stage increasing, hs-CRP levels also increased correspondingly. As shown in Table 3, eGFR has a significant negative correlation with hs-CRP and significant positive correlation with $\lg G$ and $\lg M$, whereas $\lg A$ did not show any significant correlation.

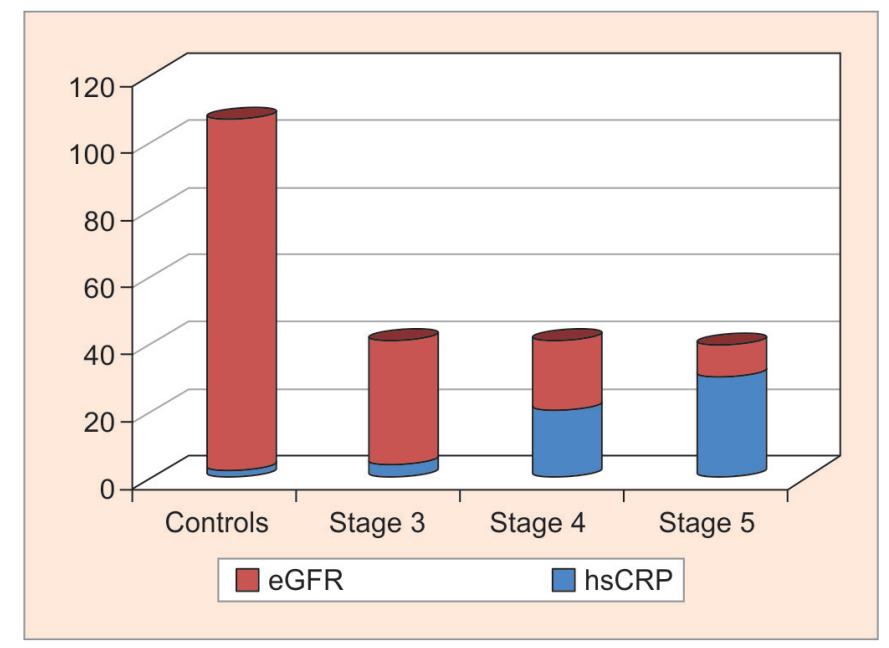

Fig. 6: Showing eGFR and hsCRP in controls and CKD stages

\section{Discussion}

Immune (dys) regulation and inflammatory activation in CKD is multifactorial; some of them may be related to the primary disease rather than to uremic state per se.

\section{CKD and Inflammation}

Low-grade inflammation is commonly seen in CKD patients even before the start of renal replacement therapy for them, and this 
Table 3: Correlation eGFR with hsCRP and Immunoglobulins.

\begin{tabular}{lll}
\hline & $e G F R$ & \\
\cline { 2 - 2 } Variables & Spearman correlation $(\rho)$ & pvalue \\
\hline hs-CRP & -0.47 & $<0.0001^{*}$ \\
$\lg G$ & 0.22 & $0.007^{*}$ \\
IgA & 0.01 & 0.89 \\
IgM & 0.43 & $<0.0001^{*}$ \\
\hline
\end{tabular}

${ }^{*} p<0.05$ is considered significant

persistent inflammation may also be a contributory risk factor for progression of CKD and vascular disease.10 When renal function declines, many factors such as retention of proinflammatory cytokines, reactive oxygen species, advanced glycation end products, autonomic dysfunctions, and volume overload may contribute to inflammation. A more thorough categorization of uremic retention solutes with regard to their specific pro- and antiinflammatory properties are required. ${ }^{10}$

In our study, we observed elevated serum hsCRP and decreased serum immunoglobins levels ( $\mathrm{gM}$ and $\lg G$ ) with a decline in renal function. The failure in kidney function was assessed by the eGFR. There was a significant association between CKD stages and hsCRP levels, though the relationship between inflammation and progression of renal function impairment in these patients is not clearly understood. Inflammation itself is considered as the main component of histological features of kidney diseases and as an independent predictor of adverse outcomes. ${ }^{11,12}$ Our study also has shown that as the eGFR is decreasing hsCRP is increasing which was statistically significant (rho-0.45, $p<0.05$ ).

\section{CKD and Immunoglobulins}

Renal failure has many infectious complications, and uremic state, itself, impairs immunity and predisposes the host to infection. The relationship between the uremic state, impaired immune status, and susceptibility to infection have never been established. ${ }^{13}$

Our study has shown decreased immunoglobulin as compared to healthy individuals as well as across the CKD. Various reasons for the decrease in immunoglobulin have been noted.

A study by Alexiewicz et al. ${ }^{14}$ explained that chronic renal failure patients have secondary hyperparathyroidism and increased blood levels of PTH. ${ }^{15-17}$ Since PTH inhibits B cell proliferation, 14 it is possible that the chronic exposure of $B$ cells in uremia to excess PTH which may affect their ability to produce immunoglobulins in response to antigenic stimulation. Such a potential effect of PTH would provide, a partial explanation for the declined humoral immunity in chronic kidney failure which supports our study findings of decreased immunoglobulins across the stages of CKD. ${ }^{18}$

There was a decrease in IgG levels in our study in the CKD stages which may be due to a loss in urine or impaired B cell maturation for its production. One study has shown that glomerular IgG deposits were independently associated with poor renal outcome IgA nephropathy patients. In addition, glomerular IgG deposition correlated with greater histological activity and increased clinical severity suggesting that these depositions may have a role in the progression of CKD. ${ }^{19}$

The deposition of Immunoglobulins G, M, A, C3, and C4 together with $\mathrm{C} 1 \mathrm{q}$ is the hallmark of lupus nephritis and is stated to as the 'full house pattern' of immune deposition. ${ }^{20}$ This pattern of deposition in kidneys may mark the decrease of immunoglobulin levels in most of these patients.
In our study, we found there is marked decrease in IgM levels in CKD patients compared to controls, and the reduction is very significant in CKD stages, as the eGFR is decreasing lgM levels also decreasing (negative correlation). Glomerular immunoglobulin M (IgM) deposition occurs in a variety of glomerular diseases, but the mechanism of deposition remains controversial. Some studies theorized that IgM becomes passively trapped in areas of glomerulosclerosis, and other recent studies found that $\lg M$ specifically binds to the damaged glomeruli. Immunofluorescence microscopy demonstrated mesangial and capillary loop deposition of IgM, whereas ultrastructural analysis found IgM deposition on endothelial cells as well as in subendothelial areas. ${ }^{21}$ Or it may be due to antiglobulin binding (whether by monoclonal rheumatoid factors, polyclonal rheumatoid factors or IgM antibodies to human immunoglobulins) depends upon the multivalency of IgM antibodies in which each of the binding sites in the Fab portion of the molecule is of low affinity, but stable binding to aggregated or complexed immunoglobulins occurs because of multipoint attachment. $^{22,23}$

\section{Conclusion}

Based on our findings it can be concluded that a deficiency of Immunoglobulins was noted in a considerable number of uremic patients from all stages of CKD, suggesting inhibition of their synthesis by the uremic state. Uremia is associated with a state of immune dysfunction characterized by immunodepression that leads to the high prevalence of infections, as well as by immune activation resulting in inflammation (increased hs-CRP). The immune system deterioration by itself or through predisposition to infections leads to inflammation which significantly contributes to high premature mortality in CKD patients. Thus, measures aimed at attenuating immune abnormalities in CKD are needed which may lead to a decrease in morbidity and mortality in these patients.

\section{Limitations}

Limitation of our study is the small sample size. And we assessed the only hs-CRP to know the inflammatory status of the patients. Patients who attended our referral hospital were already in the advanced stages of CKD; hence there was a paucity of cases in stages 1 and 2 .

\section{References}

1. Chapter 1: Definition and classification of CKD. Kidney International Supplements. 2013;3(1):19-62.

2. Agarwal SK, Srivastava RK: Chronic Kidney Disease in India: Challenges and Solutions. Nephron ClinPract 2009;111:c197-c203.

3. Indian Society of Nephrology to organize ISNCON 2017 in New Delhi. December 13, 2017, 16:22 IST. Press note.

4. Pálsson R, Patel UD. Cardiovascular Complications ofDiabetic Kidney Disease. Advances in chronic kidney disease. 2014;21(3):273-280.

5. Johnson DW, Fleming SJ. The use of vaccines in renal failure. ClinPharmacokinet. 1992 Jun; 22(6):434-446.

6. Kato S, Chmielewski M, Honda $\mathrm{H}$, et al. Aspects of Immune Dysfunction in End-stage Renal Disease. Clinical Journal of the American Society of Nephrology : CJASN. 2008;3(5):1526-1533.

7. Silverstein DM. Inflammation in chronic kidney disease: role in the progression of renal and cardiovascular disease.Pediatr Nephrol.2009 Aug;24(8):1445-1452.

8. Chen L, Deng H, Cui H, et al. Inflammatory responses and inflammationassociated diseases in organs. Oncotarget. 2018;9(6):7204-7218. 
9. Dai L, Golembiewska E, Lindholm B, Stenvinkel P.End-Stage Renal Disease, Inflammation and Cardiovascular Outcomes.Contrib Nephrol 2017;191:32-43.

10. Suliman ME, Stenvinkel P.Contribution of inflammation to vascular disease in chronic kidney disease patients.Saudi J Kidney Dis Transpl.2008 May;19(3):329-345.

11. Menon V, Greene T, Wang X, Pereira AA, Marcovina SM, et al. C-reactive protein and albumin as predictors of all-cause and cardiovascular mortality in chronic kidney disease. Kidney Int2005;68: 766-772.

12. Koeda Y, Nakamura M, Tanaka F, Onoda T, Itai K, et al. Serum C-reactive protein levels and death and cardiovascular events in mild to moderate chronic kidney disease. Int Heart J 2011; 52: 180-184.

13. Isabel A, Clarke, Douglas J. Ormrodand Thomas E. Miller. Host immune status in uremia. Effect of uremia on resistance to bacterial infection. Kidney International 1983; Vol. 24, pp. 66-73.

14. Alexiewicz JM, Klinger M, Pirrs TO, Gaciong Z, Linkerisraeli M, Massry SG: Parathyroid hormone inhibits B cell proliferation: Implications in chronic renal failure. J Am SocNephrol 1990;1: 236-244.

15. Katz AL, Hampers CL, Merrill JP: Secondary hyperparathyroidism and renal osteodystrophy in chronic renal failure. Medicine (Baltimore) 1969;48:333-374.
16. Berson SA, Yalow RS: Parathyroid hormone in plasma in adenomatous hyperparathyroidism, uremia, and bronchogenic carcinoma. Science 1966; 154:907-909.

17. Massry SG, Coburn JW, Peacock M, Kleeman CR:Turnover of endogenous parathyroid hormone in uremic patients and those undergoing hemodialysis. Trans Am SocArtif Intern Organ 1972;18:416-420.

18. Gaciong Z, Alexiewicz J, Linker-Israeli M, Shulman I, Pitts T, Massry S. Inhibition of immunoglobulin production by parathyroid hormone, implications in chronic renal failure. Kidney international. 1991;40(1):96-106.

19. Shin DH, Lim BJ, Han IM, Han SG, Kwon YE, Park KS et al. Glomerular IgG deposition predicts renal outcome in patients with IgA nephropathy. Modern Pathology 2016; 29: 743-752.

20. Stefan P. Berger AnjaRoos Mohamed R. Daha. Complement and the kidney: What the nephrologist needs to know in 2006? NephrolDial Transplant 2005; 20: 2613-2619.

21. Panzer SE, Laskowski J, Renner B, et al. IgM exacerbates glomerular disease progression in complement-induced glomerulopathy. Kidney Int. 2015;88(3):528-537.

22. Eisenberg R. The specificity and polyvalency of binding of a monoclonal rheumatoid factor. Immunochemistry 1976 Apr; 13(4):355-359.

23. Dissanayake S, Hay FC, Roitt IM. The binding constants of IgM rheumatoid factors and their univalent fragments for native and aggregated human IgG. Immunology. 1977;32(3):309-318. 